\title{
From cure to care: the development of hospice care in Taiwan
}

\section{Introduction}

Hospice care stresses a combination of high humanity and high technology, centered on treatment of the person not the disease, hospital and home care provision, with high quality holistic care for the whole person, whole family, whole process and whole team, in order to serve patients suffering from terminal illness and their families. ${ }^{1}$

\section{The development of hospice care in Taiwan}

From the document literature the hospice care movement germinated in Taiwan in 1983, when the Catholic Sanipax SocioMedical Service and Education Foundation started Terminal Cancer Patient Homecare. Healthcare workers and trained volunteers went to the homes of terminal cancer patients to deliver care services. In 1990, Mackay Memorial Hospital Tamsui Branch opened a hospice ward, and at the end of the year established the Hospice Foundation of Taiwan. In 1994 the Lotus Palliative Care Foundation was founded. The combined influence of these three foundations helped set up the Taiwan Academy of Hospice Palliative Medicine (TAHPM) in 1999, to promote the hospice movement in partnership. The year 2000 was a milestone in hospice care for Taiwan when the Legislative Yuan passed the Hospice Palliative Care Act after three readings and in the same year announced its implementation. The new law guaranteed the right to natural and dignified death for terminally ill patients in Taiwan. ${ }^{2,3}$

As early as 1995, Taiwan's Ministry of Health established a Hospice Palliative Care Promotion Team, which commissioned the Hospice Foundation of Taiwan to organize the Hospice Palliative Care Team Professional Training Program. In 1996, the Palliative Care Ward Governing Regulations and the Hospice Homecare Governing Regulations were announced, which allowed applications to the National Health Insurance Hospice Homecare Coverage Pilot Project. To fill in the gaps in hospice care, in 2000 the Health and Welfare Bureau of the Department of Health, Executive Yuan, launched a National Health Insurance Coverage of Holistic Hospice Care Pilot Project, which brought hospitalized hospice care into the experimental program to give terminal cancer patients an alternative care option to traditional treatment. In 2006, hospice treatment was officially covered by National Health Insurance. In 2009, the central government's National Health Insurance Administration announced hospice care for eight non-cancer diseases. ${ }^{2,3}$

The development of hospice palliative care in Taiwan started in 1990 with the establishment of the first hospice ward. Taiwan's diverse hospice palliative care model includes hospice wards, hospice homecare, hospice day wards, and hospital-based hospice palliative healthcare teams. Thanks to promotion by the government, NGOs, industry and academia, the number of palliative hospice care hospitals is continually increasing. According a 2017 survey of the state of hospice palliative treatment conducted by the National Health Insurance Administration of the Ministry of Health and Welfare (MOHW), there are 62 hospitals in Taiwan with hospice wards, 101
Volume 2 Issue 5 - 2018

\author{
Chun-Ying Cho \\ Department of Social Work, Chang Jung Christian University, \\ Taiwan
}

Correspondence: Chun-Ying Cho, Associate Professor, Department of Social Work, Chang Jung Christian University, Taiwan,Email cycho@mail.cjcu.edu.tw

Received: August 29, 2018 | Published: October 15, 2018

hospitals with hospice homecare programs, 200 hospitals that conduct community hospice care, and 142 hospitals that conduct collective hospice care. However, outlying islands (Kinmen, Penghu and Lian chiang Counties) do not enjoy full coverage. In August 1, 2006, registration of DNR compliant IC health insurance cards started. On September 1, 2006, registration of optional DNR IC health insurance cards started. From September 18, 2006 to March 2017, the National Health Insurance Administration, MOHW, recorded 423,956 successful applications from the public., ${ }^{4,5}$

In terms of international standards, according to the Singaporean Lien Foundation's global quality of death index, Taiwan's hospice care ranked 14th (out of 40) in the world in 2010. In 2015, Taiwan achieved the ranking of number six (out of 80 ). On both occasions Taiwan was placed first in Asia. ${ }^{4,6,7}$ However, there is still work to be done to promote hospice care on Taiwan's outlying islands.

\section{From the Hospice Palliative Care Act to Patient Autonomy Act}

After passing the Hospice Palliative Care Act in 2000, on December 18, 2015 Taiwan's Legislative Yuan passed the Patient Right to Autonomy Act in three readings, which was the first law in Asia to specifically protect the right of the patient to autonomy, and set a precedent in Taiwan for patient-centered guarantees to the right of the patient to be informed, have freedom of choice, and to make policy decisions. It returned the right to healthcare decisions and good death to the patient. The law states that anyone aged 20 years or older with full disposing capacity has priority right to be informed, choose and make decisions regarding diagnosis and treatment options. Furthermore, via Advance Care Planning and Advance Directions, when a patient meets one of five clinical conditions, he or she may choose to accept or refuse treatment. In addition, a healthcare agent designated in advance may express the opinion of a patient who is in a coma or incapable of clearly expressing their own opinion. The five clinical conditions are:
a. The patient is terminally ill.
b. The patient is in an irreversible coma.
c. The patient is in permanent vegetative state.
d. The patient is suffering from severe dementia. 
e. Other disease conditions, announced by the central competent authority, that shall meet all of the following requirements that the conditions or sufferings are unbearable, that the disease is incurable and that there are no other appropriate treatment options available given the medical standards at the time of the disease's occurrence. ${ }^{4,8,9}$

Most Taiwanese people are unfamiliar with this law on patient healthcare autonomy, and easily confuse it with the Hospice Palliative Care Act. To assist smooth transition of the law, by special regulation the law is to be implemented three years after being announced. In order to let the public understand the essence of the "Patient Autonomy Act", the Ministry of Health and Welfare has specially commissioned 7 hospitals in Taiwan to conduct trials in early 2017, so that the medical staff or social workers who standing in the front line will have the correct laws, and passed on to interested people, patient and their families.

\section{Conclusion}

The adoption of the patient autonomy Act is an important milestone of hospice care in Taiwan. Based on the need to prioritize dignified life and improve the quality of medical care, MOHW should gather ideas from the medical field, not just to build consensus but to promote relevant pilot projects. At the same time, to improve understanding among medical workers and the public, MOHW should organize educational training programs to dispel misconceptions and raise public awareness. It is essential that three years after its announcement the law can be smoothly implemented to create a winwin situation for medical healthcare. ${ }^{5,10}$ Hospice medical care has been on Taiwan's statute books for 17 years, and in 2015 the Patient Right to Autonomy Act was passed. For critically ill patients from active treatment to hospice palliative care. However, euthanasia continues to be a contentious topic. Wang Xiao min, who was bedridden in a persistent vegetative state for half a century due to a road accident, and finally passed away in 2010 at the age of 64, was the first case to apply for euthanasia in Taiwan. At the time, 17-year-old Wang was a high school student and the conductor of her school marching band. On the way to school, she was hit by a taxi and fell into a persistent vegetative state. Burdened with caring for her until her death, Wang's mother, when she was alive, persistently asked for more attention to be paid to the issue of euthanasia, which resulted in reform of the Hospice Palliative Care Act, marking the first step to legalizing euthanasia. ${ }^{10}$

In addition, in 2017, when well-known 84-year-old former sports broadcaster Fu Da-jen contracted pancreatic cancer and was given just two months to live, he wrote to the President to advocate for a euthanasia law. Celebrated writer Chung Yao also called for a euthanasia law after her husband was physically and mentally incapacitated by a stroke. The two celebrities lobbied together for a euthanasia law, saying, "only legislative action can reduce the large number of living dead in Taiwan". ${ }^{11}$ In Taiwan, because euthanasia is a moral, medical and legal issue, which is also bound up with value judgements, there is still a long way to go before society can reach the consensus that would be a precondition for further relevant legislation.

\section{Acknowledgements}

None.

\section{Conflict of interest}

The author declares no conflict of interest.

\section{References}

1. Chao CS. Development and prospects of hospice palliative care in Taiwan. Hu Li Za Zhi. 2009;56(1):5-10.

2. Chen RC. A personal journey in Taiwan's hospice palliative care movement. BAOJ Pall Medicine. 2016;2:019.

3. Lin Ming-Hui, Chen Tzeng-Ji. Current developments in hospice treatment in Taiwan. Taiwan Cancer Prevention Network. 2009.

4. Chen RC. Hospice treatment philosophy, history, working models, current status and future prospects. Lien Foundation spiritual care curriculum. 2017.

5. National Health Insurance Administration, Ministry of Health and Welfare. 2017

6. Lien Foundation. The quality of death, Ranking end-of-life care across the world. Economist Intelligence Unit. The Economist. 2010:1-36.

7. Lien Foundation. The quality of death, Ranking end-of-life care across the world. Economist Intelligence Unit. The Economist. 2015:1-71.

8. Chen RC. How the Patient Right to Autonomy Act can maintain good death for patients in persistent vegetative states. Health World. 2016;470:3-5

9. Zhang Jing Hui. After passing the patient right to autonomy act, good death is still 4 steps away. Common health Magazine. 2016:207.

10. NOW News 2011/01/05 00:23. Bedridden for half a century, vegetative state Wang Xiao min dies March 2010.

11. Liberty Times 2017-10-11 17:21. Fu Da-jen: I don’t want Taiwan to allow living death. 